\title{
CCR7 as a predictive biomarker associated with computed tomography for the diagnosis of lymph node metastasis in bladder carcinoma
}

\author{
JINBO CHEN* ${ }^{*}$ YU CUI*, LONGFEI LIU, CHAO LI, YUNHUA TANG, XU ZHOU, LIN QI and XIONGBING ZU \\ Department of Urology, Xiangya Hospital, Central South University, Changsha, Hunan 410008, P.R. China
}

Received December 5, 2014; Accepted October 19, 2015

DOI: $10.3892 / \mathrm{ol} .2015 .3939$

\begin{abstract}
The aim of the present study was to investigate whether the expression levels of CC-chemokine receptor 7 (CCR7) combined with computed tomography (CT) was associated with lymph node metastasis in bladder transitional cell carcinoma (BTCC). For this purpose, 115 cases of BTCC were analyzed at the Department of Urology of Xiangya Hospital, Central South University (Changsha, China). Preoperative CT scans of abdomen and pelvis, immunohistochemistry of CCR7 expression in the tumor specimens and pathological findings for lymph node metastasis were assessed. In addition, the sensitivity, specificity and accuracy of CCR7 and CT for the diagnosis of lymph node metastasis in BTCC were evaluated separately and jointly. The expression levels of CCR7 were observed to be significantly higher in BTCC than in normal controls $(\mathrm{P}<0.01)$. Multivariate analysis indicated that the overexpression of CCR7 was an independent predictor for lymph node metastasis in BTCC $(\mathrm{P}<0.05)$. The sensitivity, specificity and accuracy of CCR7 combined with CT scan for the diagnosis of lymph node metastasis in BTCC were 92.3, 83.6 and $70.0 \%$, respectively. By contrast, the sensitivity, specificity and accuracy of CCR7 alone were 88.1,69.9 and 76.5\%, respectively, while the sensitivity, specificity and accuracy of CT alone were 52.4, 79.5 and $69.6 \%$, respectively. The results of the present study indicated that CCR7 is an independent predictor of lymph node metastasis in BTCC. Therefore, the
\end{abstract}

Correspondence to: Professor Xiongbing $\mathrm{Zu}$, Department of Urology, Xiangya Hospital, Central South University, 78 Xiang Ya Road, Changsha, Hunan 410008, P.R. China

E-mail:whzuxb@163.com

*Contributed equally

Abbreviations: CCR7, CC-chemokine receptor 7; CT, computed tomography; BTCC, bladder transitional cell carcinoma; PUNLMP, papillary urothelial neoplasm of low malignant potential; LGPUC, low-grade urothelial carcinoma; HGPUC, high-grade urothelial carcinoma

Key words: CCR7, computed tomography, bladder carcinoma, lymph node metastasis use of CCR7 combined with CT may improve the accuracy of the diagnosis of lymph node metastasis in BTCC.

\section{Introduction}

Bladder transitional cell carcinoma (BTCC) is one of the most common types of malignant tumor of the urinary system. The treatment of BTCC is challenging, due to its high occurrence of metastasis (1). Survival in muscle-invasive BTCC depends mainly on the pathological stage of the disease (2), and lymph node metastasis is currently considered to be the most highly-dependent variable associated with survival (3).

Evidence from previous studies suggests that chemokines and their receptors participate in tumor metastasis (4). CC-chemokine receptor 7 (CCR7) is naturally a homeostatic chemokine receptor expressed by various subtypes of immune cells that migrate to and within lymphoid organs (5). Previous studies have demonstrated that the overexpression of CCR7 in tumor cells is significantly associated with lymph node metastasis in gastric, non-small cell lung, breast and bladder cancer (6,7). Spiral computed tomography (CT) is commonly used to evaluate lymph metastasis in bladder cancer. However, the sensitivity and accuracy of CT is limited (8). A previous study by Li et al (9) reported that the use of CT combined with vascular endothelial growth factor $\mathrm{C}$ may improve the accuracy of the diagnosis of lymph node metastasis in bladder carcinoma.

Therefore, in the present study, the potential role of CCR7 as an independent predictor for lymph node metastasis in BTCC was investigated. Furthermore, the sensitivity, specificity and accuracy of CCR7 and CT for the diagnosis of lymph node metastasis were evaluated separately and jointly. The results indicated that CCR7 is a predictive biomarker associated with CT for the diagnosis of lymph node metastasis in BTCC.

\section{Materials and methods}

Patients and treatment. The present study included 115 patients with BTCC who underwent laparoscopic radical cystectomy and pelvic lymphadenectomy in the Department of Urology of Xiangya Hospital, Central South University (Changsha, China) between January 2010 and December 2013. The study was approved by the ethics committee at Xiangya Hospital, Central South University. Written informed consent was obtained from 
all the participants in the study. The patients included in the study had undergone radical surgery of the bladder, surrounding fat tissue, remote end of the ureter and pelvic lymphadenectomy. The cranial, lateral and caudal borders of the pelvic lymphadenectomy were the level of the inferior mesenteric artery, genitofemoral nerve and pelvic floor, respectively.

The BTCC tissue specimens obtained following the above surgical procedure were fixed in $10 \%$ formaldehyde solution for postoperative histopathological examination. Normal urothelium tissue specimens were obtained from ten patients with benign prostatic hypertrophy via transurethral resection of the bladder. The histological cell type of the BTCC specimens was determined according to the 2004 World Health Organization (10) classification by an experienced pathologist who did not possess any prior knowledge of the patient's disease data. A total of 14, 42 and 59 specimens were classified as papillary urothelial neoplasm of low malignant potential, low-grade urothelial carcinoma and high-grade urothelial carcinoma, respectively. Tumor staging was reviewed based on the 2009 Union for International Cancer Control-tumor node metastasis staging system (11). Accordingly, 7, 26, 58 and 24 tumors were staged T1, T2, T3 and T4, respectively.

Immunohistochemical staining. Immunohistochemical staining of CCR7 was performed using a ready-to-use SP kit (Zhongshan Jinqiao Biotechnology Co., Ltd., Beijing, China), according to the manufacturer's protocol. Briefly, the $4-\mu \mathrm{m}$ paraffin-embedded tissue sections were subjected to deparaffinization and rehydration prior to be immersed in $0.01 \mathrm{M}$ citric buffer $(\mathrm{pH} 6.0)$. The slides were then incubated with $3 \%$ hydrogen peroxide for $10 \mathrm{~min}$, followed by incubation in normal goat serum for $10 \mathrm{~min}$ at room temperature. Anti-CCR7 rabbit monoclonal immunoglobulin (Ig)G antibody (catalogue no. ab32527; Abcam, Cambridge, MA,USA) was used at a dilution of 1:200 as primary antibody. Next, the sections were incubated with goat anti-rabbit polyclonal antibody for $10 \mathrm{~min}$, and subsequently exposed to streptavidin peroxidase (Zhongshan Jianqaio Biotechnology) for $10 \mathrm{~min}$. The slides were visualized with 3,3'-diaminobenzidine (Zhongshan Jianqaio Biotechnology), counterstained with hematoxylin (Auragene, Changsha, China), prior to be examined by microscopy (Olympus BH2; Olympus Corporation, Tokyo, Japan). For the negative controls, the primary antibody was substituted for phosphate-buffered saline.

Under a low-power microscopy field, each slide was evaluated in ten randomly selected areas that contained tumor cells, ensuring that $\geq 100$ tumor cells/field were examined. The evaluation was conducted by two independent investigators, who were unaware of the clinicopathological data. Two scoring systems were used, including staining intensity and percentage of positive cells. The staining intensity was scored on a semiquantitative 4-points scale as follows: i) A score of 0 was assigned to the negative control; ii) a score of 1 indicated weak cytoplasmic and nuclear staining, which was slightly darker than the negative control; iii) a score of 2 was indicative of moderate staining, defined as an intensity exhibiting a score of 1-3; and iv) 3 corresponded to strong staining, which was equivalent to or darker than the positive control. The percentage of stained cells was also scored on a semiquantitative 4-points scale as follows: i) 0 indicated $<10 \%$ positive cells; ii) 1 was assigned if the percentage of positive cells in the sample was
$10-25 \%$; iii) 2 corresponded to $25-50 \%$ positive cells present in the sample; and iv) 3 was assigned to those samples displaying $>50 \%$ positive cells. The staining intensity and percentage of stained cells scores were then combined as follows: i) Those specimens presenting a total score of $0-1$ were assigned a value of I; ii) II was assigned to those specimens with a total score of 2; iii) III indicated a total score of 3-4; and iv) IV was assigned to samples exhibiting a total score of 5-6.

CT evaluation. All patients included in the study had undergone CT scanning of the abdomen and pelvis $\geq 5$ days prior to cystectomy and pelvic lymphadenectomy, which had been conducted on SOMATOM Sensation 64 eco (Siemens AG, Munich, Germany), with a slice thickness and layer of $5 \mathrm{~mm}$. As contrast agent, $80 \mathrm{ml}$ Ultravist $^{\circledR}$ (Bayer AG, Leverkusen, Germany) was used. The CT images were reevaluated by urologists and uroradiologists who were unaware of the final pathological results. A pelvic lymph node $>10 \mathrm{~mm}$ in size was considered as positive lymph node metastasis.

Statistical analysis. Statistical analyses were performed using SPSS software, version 13.0 (SPSS, Inc., Chicago, IL, USA). The association between the variables was assessed by $\chi^{2}$ test, Fisher's exact probability test, Mann-Whitney U test or stepwise logistic regression analysis. Logistic regression analysis was employed for univariate analysis, while multiple logistic regressions were used for multivariate analysis. $\mathrm{P}<0.05$ was considered to indicate a statistically significant difference.

\section{Results}

CCR7 levels in patients with BTCC and normal controls. Expression of CCR7 was detected in the cytoplasm and membrane of tumor cells, but low or no staining for CCR7 was observed in the normal transitional epithelium (Fig. 1). The frequency of detectable CCR7 in the BTCC samples was markedly higher than in the normal bladder tissues $(\mathrm{P}<0.01$; Table I).

Association of CCR7 with lymph node metastasis in BTCC. In the positive lymph node metastasis group, 0, 5, 14 and 23 samples displayed expression of CCR7 of score I, II, III and IV, respectively. By contrast, in the negative lymph node metastasis group, the number of cases displaying expression levels of CCR7 of score I, II, III and IV were 15, 36, 22 and 0, respectively. These results indicated that the overexpression of CCR7 was significantly associated with pelvic lymph node metastasis $(\mathrm{P}<0.05$; Table II $)$.

Association of BTCC lymph node metastasis with clinicopathological features. BTCC pelvic lymph node metastasis was closely associated with certain clinicopathological features of the patients, including tumor size, pathological grade, clinical stage, recurrence and expression levels of CCR7. Among these, overexpression of CCR7 was identified as an independent indicator for lymph node metastasis in multivariate analysis, based on the results of stepwise logistic regression analysis (Table III).

Separate and joint evaluation of CCR7 and CT scan for the detection of BTCC lymph node metastasis. Based on the expression levels of CCR7, the samples were classified 

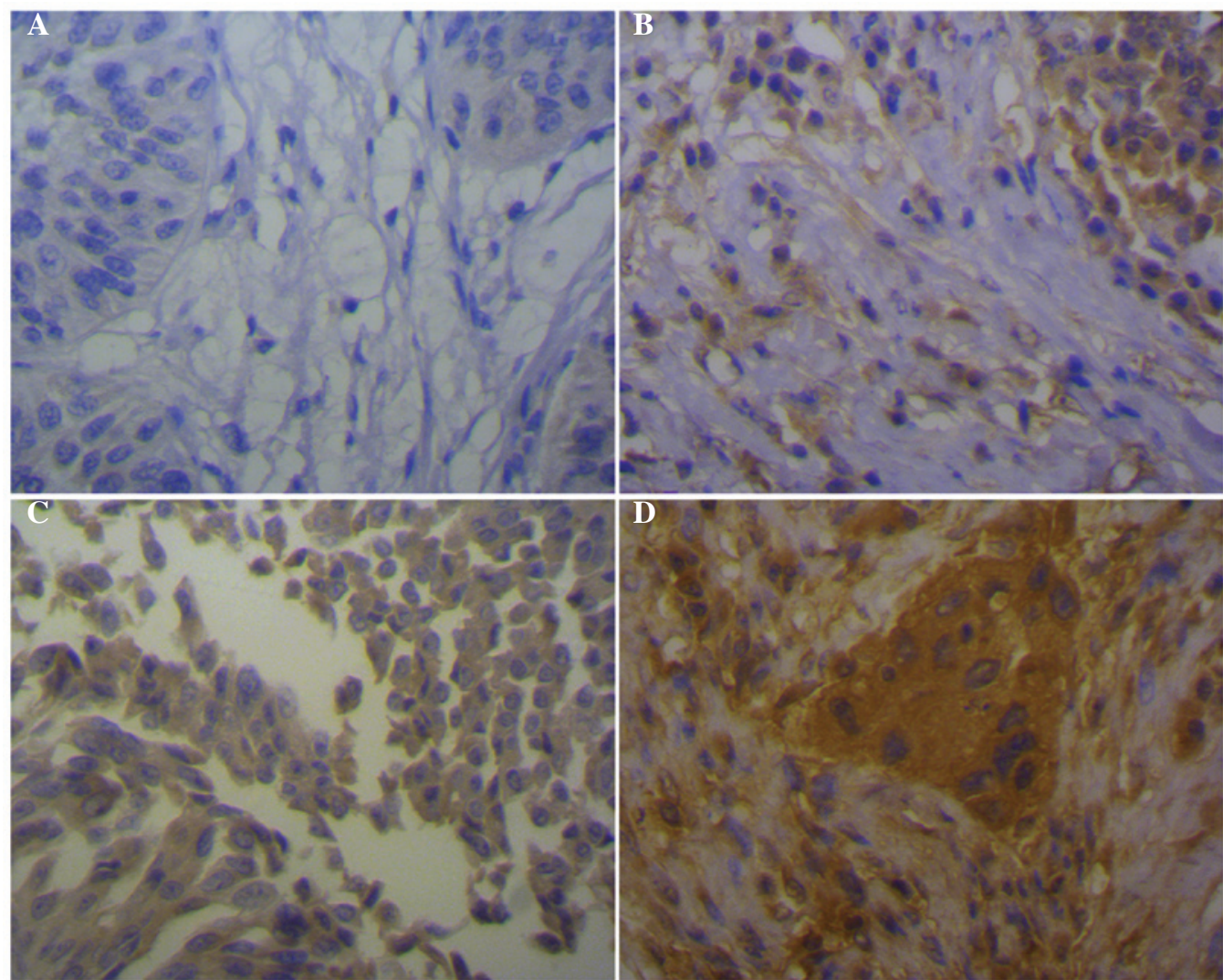

Figure 1. Immunohistochemical staining of CCR7 in bladder transitional cell carcinoma tissues. (A) Negative staining, indicating no expression of CCR7 (score I). (B) Weak positive staining, indicating low expression levels of CCR7 (score II). (C) Moderate positive staining, indicating moderate expression levels of CCR7 (score III). (D) Strong positive staining, indicating high expression levels of CCR7 (magnification, x400). CCR7, CC-chemokine receptor 7.

Table I. Expression levels (score I-IV) of CC-chemokine receptor 7 in patients with bladder transitional cell carcinoma and normal controls.

\begin{tabular}{lrrrrr}
\hline & \multicolumn{3}{c}{ CC-chemokine receptor 7 (no.) } & \\
\cline { 2 - 5 } Tissues (no. of patients) & I & II & III & IV & P-value \\
\hline Bladder transitional cell carcinoma (115) & 16 & 29 & 48 & 22 & $<0.01$ \\
Normal (10) & 8 & 2 & 0 & 0 & - \\
\hline
\end{tabular}

Table II. Association between the expression levels (score I-IV) of CC-chemokine receptor 7 and lymph node metastasis in bladder transitional cell carcinoma.

\begin{tabular}{lrrrrrr}
\hline & \multicolumn{4}{c}{ CC-chemokine receptor 7 (no.) } & \\
\cline { 2 - 5 } Lymph node metastasis (no. of patients) & I & II & III & IV & P-value \\
\hline Positive (42) & 0 & 5 & 14 & 23 & $<0.05$ \\
Negative (73) & 15 & 36 & 22 & 0 & - \\
\hline
\end{tabular}

into two groups, according to their score levels. Thus, CCR7 scores of I and II were included in the low expression or negative group, whereas CCR7 scores of III and IV corresponded to the high expression or positive group. Among the 115 cases with BTCC analyzed, 37 displayed CCR7 scores of III-IV and lymph node metastasis; 22 displayed CCR7 scores of III-IV but no lymph node metastasis; 51 exhibited CCR7 scores of I-II but no lymph node metastasis; and 5 presented CCR7 scores of I-II in addition to lymph node metastasis. The sensitivity, specificity and accuracy of CCR7 in the evaluation of lymph node metastasis were 88.1, 69.9 and $76.5 \%$, respectively. 
Table III. Univariate and multivariate analyses for bladder transitional cell carcinoma lymph node metastasis.

\begin{tabular}{|c|c|c|c|c|c|}
\hline \multirow[b]{2}{*}{ Variable } & \multicolumn{3}{|c|}{ Univariate } & \multicolumn{2}{|l|}{ Multivariate } \\
\hline & Comparison & OR $(95 \% \mathrm{CI})$ & $\mathrm{P}$-value & OR $(95 \% \mathrm{CI})$ & P-value \\
\hline Age (years) & $<60$ or $\geq 60$ & - & $>0.05$ & - & - \\
\hline Tumor size (cm) & $<3$ or $\geq 3$ & - & $>0.05$ & - & - \\
\hline Pathological grade (WHO 2004) & PUNLMP-LGPUC or HGPUC & $0.409(0.061-0.813)$ & $<0.05$ & - & $>0.05$ \\
\hline Clinical stage (UICC-TNM) & $\mathrm{T} 1-\mathrm{T} 2$ or $\mathrm{T} 3-\mathrm{T} 4$ & $0.233(0.042-0.671)$ & $<0.01$ & - & $>0.05$ \\
\hline Detection (time) & First or recurrence & - & $>0.05$ & - & - \\
\hline CCR7 expression levels (score) & I-II or III-IV & $3.860(1.710-7.590)$ & $<0.01$ & $2.930(1.650-6.710)$ & $<0.05$ \\
\hline
\end{tabular}

OR, odds ratio; CI, confidence interval; WHO, World Health Organization; PUNLMP, papillary urothelial neoplasm of low malignant potential; LGPUC, low-grade urothelial carcinoma; HGPUC, high-grade urothelial carcinoma; UICC, Union for International Cancer Control; TNM, tumor node metastasis; CCR7, CC-chemokine receptor 7.

Table IV. Sensitivity, specificity and accuracy of CCR7 alone, CT alone and CCR7 combined with CT for the diagnosis of bladder transitional cell carcinoma lymph node metastasis.

Lymph node metastasis (no. of patients)

\begin{tabular}{|c|c|c|c|c|c|}
\hline Predictor & Positive & Negative & Sensitivity (\%) & Specificity (\%) & Accuracy (\%) \\
\hline CCR7 & & & 88.1 & 69.9 & 76.5 \\
\hline Positive & 37 & 22 & & & \\
\hline Negative & 5 & 51 & & & \\
\hline CT & & & 52.4 & 79.5 & 69.6 \\
\hline Positive & 22 & 15 & & & \\
\hline Negative & 20 & 58 & & & \\
\hline CCR7 plus CT & & & 92.3 & 83.6 & 87.0 \\
\hline Positive & 39 & 12 & & & \\
\hline Negative & 3 & 61 & & & \\
\hline
\end{tabular}

CCR7, CC-chemokine receptor 7; CT, computed tomography.

CT examination revealed 37 patients with lymph node metastasis, including 29 in stage T3 and 8 in stage T4. The positive rate was $32.2 \%(37 / 115)$. Positive $\mathrm{CT}$ and presence of lymph node metastasis were observed in 22 patients, while 15 patients presented positive CT but negative lymph node metastasis, 20 displayed negative CT but positive lymph node metastasis, and 58 exhibited negative $\mathrm{CT}$ and absence of lymph node metastasis. The sensitivity, specificity and accuracy of $\mathrm{CT}$ in the evaluation of lymph node metastasis were 52.4, 79.5 and $69.6 \%$, respectively.

When CCR7 was combined with CT scan in the detection of lymph node metastasis, the sensitivity, specificity and accuracy were $92.3 \%$ (39/42 cases), 83.6\% (61/73 cases) and 87.0\% (100/115 cases), respectively (Table IV).

\section{Discussion}

Despite remarkable advances in the treatment of bladder cancer, the morbidity and mortality rates for this disease remain high. In USA, the estimated incidence and number of mortalities associated with bladder cancer in 2012 were 73,510 and 14,880 cases, respectively (12). Thus, the identification of the risks behind disease recurrence and mortality in bladder cancer is critical for adequate surveillance and selection of adjuvant therapies $(13,14)$. Numerous studies have previously investigated potential prognostic factors for patients with bladder cancer, in order to guide therapeutic approaches and improve survival outcomes (15). Lymph node metastasis was previously identified as an important prognostic factor in patients with BTCC, since the survival rate of patients with lymph node metastasis of bladder cancer is usually low (16).

Chemokines are small secreted proteins that may contribute to the regulation of leukocyte adherence to endothelial cells, leukocyte migration through transendothelial membranes and tissue invasion (17). Previous studies revealed that the binding of chemokines to their specific receptors led to the activation of numerous signaling pathways involved in the growth and metastasis of tumor cells (18). CCR7, which was formerly considered to be an orphan receptor, has recently been identified as a chemokine receptor for chemokine (C-C motif) ligand 12, 
which is expressed in several human cell lines and vascular endothelial cells (19). The expression of CCR7 on tumor cells has mainly been reported for breast, non-small cell lung, prostate, head and neck, stomach and colorectal cancer, in addition to melanoma, chronic lymphocytic and $\mathrm{T}$ cell leukemia and non-Hodgkin's lymphoma (20). Yates et al (21) demonstrated that the expression of CCR7 in bladder carcinoma cells regulated processes such as proliferation, chemotactic motility and formation of signaling complexes in these cells and the expression levels of CCR7 were observed to be upregulated in bladder tumor tissues and exfoliated urothelial cells present in the urine of patients with bladder carcinoma, which was associated with prognosis

According to previous studies, the overexpression of CCR7 appears to be implicated in lymph node metastasis in patients with esophageal, pancreatic, gastric and breast cancer, and demonstrated that the overexpression of CCR7 was an independent factor of lymph node metastasis in these patients (22-24). In the present study, CCR7 was observed to be highly expressed in BTCC tissues, compared with normal bladder tissues. In addition, the frequency of detectable CCR7 expression was observed to be significantly higher in patients with pelvic lymph node metastasis, which is consistent with previous data reported in the literature (21). Furthermore, the logistic regression analysis conducted in the present study identified the overexpression of CCR7 as an independent factor influencing pelvic lymph node metastasis in BTCC.

$\mathrm{CT}$ is commonly used to evaluate bladder cancer and provide information on lymph node metastasis. However the sensitivity and accuracy of diagnosing lymph node metastasis remains poor (8). In previous studies, Ficarra et al (25) diagnosed pathological lymph node involvement in 45 patients, but CT only identified 19 of these patients. CT appears to be limited to the detection of metastatic lymph nodes of $\geq 0.5 \mathrm{~cm}$ in size. In the present study, postoperative pathological examination results were used as standards, and the sensitivity, specificity and accuracy of CT for the evaluation of lymph node metastasis were calculated to be $52.4,79.5$ and $69.6 \%$, respectively, which is somewhat in agreement with previous studies. These results confirm the limitations of CT for detecting lymph node metastasis in bladder carcinoma. Thus, a more sensitive method to detect lymph node metastasis at early stage is required.

In the present study, the diagnosis of lymph node metastasis using CCR7 identified 42 cases that were positive for lymph node metastasis, 37 of which were confirmed to be correct. In 56 cases that appeared to be absent of lymph node metastasis based on their expression levels of CCR7, 5 presented lymph nodes metastasis, according to pathological diagnosis. The sensitivity, specificity and accuracy of CCR7 in the evaluation of lymph node metastasis were observed to be 88.1, 69.9 and $76.5 \%$, respectively. Thus, the sensitivity of CCR7 appeared to be higher than that of CT (88.1 vs. $52.4 \%$, respectively), but its specificity was lower (69.9 vs. $79.5 \%)$. Therefore, the expression levels of CCR7 were combined with the results of CT scan, and the combined sensitivity increased to $92.3 \%$ (39/42 cases), whereas the combined specificity and accuracy were determined to be $83.6 \%$ (61/73 cases) and $87.0 \%$ (100/115 cases, respectively). Thus, a combination of CT and CCR7 detection may improve the sensitivity of the diagnosis of lymph node metastasis in BTCC.
In conclusion, the results of the present study suggest the potential clinical application of CCR7 as a predictive biomarker for diagnosing lymph node metastasis in BTCC. Furthermore, the combined evaluation of CCR7 and CT appears to be a more reliable marker for lymph node metastasis in BTCC, compared to the diagnosis by CT or CCR7 alone. These results require further confirmation by large sample and multi-center prospective studies.

\section{Acknowledgements}

The present study was supported by the National Natural Science Foundation of China (Beijing, China) (grant no. 30700832) and the Fundamental Research Funds for the Central Universities of Central South University (Changsha, China) (grant no. 72150050586).

\section{References}

1. Kaufman DS, Shipley WU and Feldman AS: Bladder cancer. Lancet 374: 239-249, 2009.

2. Stein JP, Lieskovsky G, Cote R, Groshen S, Feng AC, Boyd S, Skinner E, Bochner B, Thangathurai D, Mikhail M, et al: Radical cystectomy in the treatment of bladder cancer: Long-term results in 1,054 patients. J Clin Oncol 19: 666-675, 2001.

3. Herr HW: Superiority of ratio based lymph node staging for bladder cancer. J Urol 169: 943-945, 2003.

4. Sarvaiya PJ, Guo D, Ulasov I, Gabikian P and Lesniak MS Chemokines in tumor progression and metastasis. Oncotarget 4: 2171-2185, 2013.

5. Comerford I, Harata-Lee Y, Bunting MD, Gregor C, Kara EE and McColl SR: A myriad of functions and complex regulation of the CCR7/CCL19/CCL21 chemokine axis in the adaptive immune system. Cytokine Growth Factor Rev 24: 269-283, 2013.

6. Cassier PA, Treilleux I, Bachelot T, Ray-Coquard I, Bendriss-Vermare $\mathrm{N}$, Ménétrier-Caux $\mathrm{C}$, Trédan $\mathrm{O}$, Goddard-Léon S, Pin JJ, Mignotte H, et al: Prognostic value of the expression of C-Chemokine receptor 6 and 7 and their ligands in non-metastatic breast cancer. BMC Cancer 11: 213, 2011.

7. Cunningham HD, Shannon LA, Calloway PA, Fassold BC, Dunwiddie I, Vielhauer G, Zhang M and Vines CM: Expression of the $\mathrm{C}-\mathrm{C}$ chemokine receptor 7 mediates metastasis of breast cancer to the lymph nodes in mice. Transl Oncol 3: 354-361, 2010.

8. Baltaci S, Resorlu B, Yagci C, Turkolmez K, Gogus C and Beduk Y: Computerized tomography for detecting perivesical infiltration and lymph node metastasis in invasive bladder carcinoma. Urol Int 81: 399-402, 2008.

9. Li Z, Qi F, Miao J, Zu X, He W, Wang L and Qi L: Vascular endothelial growth factor-C associated with computed tomography used in the diagnosis of lymph node metastasis of bladder carcinoma. Arch Med Res 41: 606-610, 2010.

10. Montironi R and Lopez-Beltran A: The 2004 WHO classification of bladder tumors: A summary and commentary. Int J Surg Pathol 13: 143-153, 2005.

11. Cheng L, Montironi R, Davidson DD and Lopez-Beltran A Staging and reporting of urothelial carcinoma of the urinary bladder. Mod Pathol 22: S70-95, 2009.

12. Siegel R, Naishadham D and Jemal A: Cancer statistics, 2012. CA Cancer J Clin 62: 10-29, 2012.

13. Kim H, Kim M, Kwak C, Kim HH and Ku JH: Prognostic significance of lymphovascular invasion in radical cystectomy on patients with bladder cancer: A systematic review and meta-analysis. PLoS One 9: e89259, 2014.

14. Zaravinos A, Volanis D, Lambrou GI, Delakas D and Spandidos DA: Role of the angiogenic components, VEGFA, FGF2, OPN and RHOC, in urothelial cell carcinoma of the urinary bladder. Oncol Rep 28: 1159-1166, 2012.

15. Kamat AM, Vlahou A, Taylor JA, Hudson ML, Pesch B, Ingersoll MA, Todenhöfer T, van Rhijn B, Kassouf W, Barton Grossman $\mathrm{H}$, et al: Considerations on the use of urine markers in the management of patients with high-grade non-muscle-invasive bladder cancer. Urol Oncol 32: 1069-1077, 2014. 
16. Türkölmez K, Tokgöz H, Reşorlu B, Köse K and Bedük Y: Muscle-invasive bladder cancer: Predictive factors and prognostic difference between primary and progressive tumors. Urology 70: 477-481, 2007.

17. Kakinuma T and Hwang ST: Chemokines, chemokine receptors, and cancer metastasis. J Leukoc Biol 79: 639-651, 2006.

18. Homey B, Müller A and Zlotnik A: Chemokines: Agents for the immunotherapy of cancer? Nat Rev Immunol 2: 175-184, 2002.

19. Hao M, Zheng J, Hou K, Wang J, Chen X, Lu X, Bo J, Xu C, Shen K and Wang J: Role of chemokine receptor CXCR7 in bladder cancer progression. Biochem Pharmacol 84: 204-214, 2012.

20. Zlotnik A, Burkhardt AM and Homey B: Homeostatic chemokine receptors and organ-specific metastasis. Nat Rev Immunol 11: 597-606, 2011.

21. Yates TJ, Knapp J, Gosalbez M, Lokeshwar SD, Gomez CS Benitez A, Ekwenna OO, Young EE, Manoharan M and Lokeshwar VB: C-X-C chemokine receptor 7: A functionally associated molecular marker for bladder cancer. Cancer 119: 61-71, 2013
22. Irino T, Takeuchi H, Matsuda S, Saikawa Y, Kawakubo H, Wada N, Takahashi T, Nakamura R, Fukuda K, Omori T and Kitagawa Y: CC-Chemokine receptor CCR7: A key molecule for lymph node metastasis in esophageal squamous cell carcinoma. BMC Cancer 14: 291, 2014.

23. Lee HJ, Song IC, Yun HJ, Jo DY and Kim S: CXC chemokines and chemokine receptors in gastric cancer: From basic findings towards therapeutic targeting. World J Gastroenterol 20: 1681-1693, 2014.

24. Guo J, Lou W, Ji Y and Zhang S: Effect of CCR7, CXCR4 and VEGF-C on the lymph node metastasis of human pancreatic ductal adenocarcinoma. Oncol Lett 5: 1572-1578, 2013.

25. Ficarra V, Dalpiaz O, Alrabi N, Novara G, Galfano A and Artibani W: Correlation between clinical and pathological staging in a series of radical cystectomies for bladder carcinoma. BJU Int 95: 786-790, 2005. 